\title{
Die richtige Bewegungsform der Membrana basilaris.
}

(Fortsetzung von: „Die Uebertragung der Energie u. s. w.")

Von

\section{Emile ter Kuile.}

(Hierzu Tafel IV und 1 Textfigur.)

XIII. Erste denkbare Form (Fig. 1).

Es könnte sein, dass die Membran wirklich genau so eine Bewegung hätte, wie ich in meinem vorigen Beitrag beschrieben habe: durch die Lympheverschiebung wird die Grundmembran in der Form eines Kreissegmentes ausgebuchtet, beim Hineingepresstwerden (= Stossbewegung) des Steigbügels nach unten: beim Herausgezogenwerden ( = Zugbewegung) nach oben. Bei der Ausbuchtung nach unten wird, wie aus der Fig. 1 ersichtlich, die Netzmembran nach aussen verschoben, der nach dem Lab. vestibulare hin gerichteten Spannung im inneren Pfeiler wird entgegengewirkt; bei der Zurückbewegung und der Ausbuchtung nach oben geschieht das Umgekehrte, die nach dem Lab. vertibulare gerichtete Spannung des inneren Pfeilers sorgt dafür, dass die Membrana reticularis so viel nach innen verschoben wird, als die Bewegung des Fusses des äusseren Pfeilers nach oben solches gestattet. Es wird hierbei also angenommen, dass die erwähnte Spannung im inneren Pfeiler nicht so gross ist, dass sie die Bewegungsform der Grundmembran erheblich beeinflusst.

XIV. Die Amplitude.

Bei der beschriebenen Bewegungsform wird sich die grösste Amplitude in der Mitte $M$ (Fig. 1) finden. Die Grösse der Verschiebung der Membrana reticularis $B P=B B_{1} \cos \Varangle P B B_{\mathbf{1}}$. $B B_{1}$ wird zunächst abhängen von dem Verlältniss $\frac{B C}{A C}$. Die Vergrősserung von $A A_{1}$ der Amplitude des Fusspunktes des äusseren Pfeilers auf $B B_{1}$ ist un so beträchtlicher, je mehr $B C$ grösser ist 
als $A C$. Hierin ist $B C$ die Länge des inneren Pfeilers, $A C$ der $A b$ stand der Pfeilerfüsse. $A A_{1}$ ist aber desto grösser, je näher $A$ zu $M$ liegt; wenn $\frac{C A}{C M}$ oder auch $\frac{C A}{C E}$ (worin $C E$ die Breite der Grundmembran) grösser wird, nimmt auch $A A_{1}$ zu. Die Grössen $C A$ und $C E$ variiren nach den Messungen von Hensen derart, dass beide zunehmen vom runden Fenster bis zum Hamulus. Der Kürze wegen nenne ich nach dem runden Fenster hin: proximal, nach dem Hamulus hin: distal. Die Grösse $\frac{C A}{C E}$ variirt von proximal nach distal von

$$
\frac{0,019}{0,04125} \text { bis zu } \frac{0,085}{0,495}
$$

d. h. dass die Grösse $\frac{C A}{C E}$ proximal ungefähr 2,7 mal so gross ist als distal. Dies bringt für einen bestimmten Werth von $M M_{1}$ ein Grössersein von $A A_{1}$ proximal, ein Kleinersein distal. Wenn $A A_{1}$ grösser ist, ist auch $B B_{1}$ grösser.

XV. Ferner wird $B B_{1}$ ebenfalls um so grösser sein, je nachdem $\frac{B C}{A C}$ grösser ist. Dieses Verhältniss variirt von proximal nach distal von

$$
\frac{0,048}{0,019} \text { auf } \frac{0,085}{0,085} \text {. }
$$

Auch $\frac{B C}{A C}$ ist also proximal grösser und zwar $\pm 2,5$ mal so gross wie distal. Es liegen also zwei Momente vor, die für einen bestimmten Werth von $M M_{1}$ die Verschiebung des Kopfes des inneren Pfeilers und der Netzmembran, also die Amplitude der Haarzellenhärchen, proximal grösser sein lassen als distal. Wie aus den absoluten Werthen von $\frac{C A}{C M}$ und $\frac{B C}{C A}$ wohl ersichtlich, würde am runden Fenster sogar eine Vergrösserung, am Hamulus dagegen eine beträchtliche Verkleinerung der Amplitude stattfinden. - Würden wir die Helmholtz'sche Theorie annehmen, so würde diese Thatsache im Einklang damit sein, dass die hohen Töne mit ihren kleinen proximal, die tiefen Töne mit ihren grossen Amplituden distal percipirt werden. Wenn wir jedoch tiefer in die Sache eindringen, so sehen wir, dass, wie wir auch die Verhältnisse auszubeuten versucht haben, ein ganz tiefer Ton von derselben Intensität wie ein ganz hoher den Härchen eine so ungeheuer viel grössere Amplitude mit- 
- theilen würde, dass, wenn dieselbe bei dem hohen Ton auch noch so klein wäre, die Zellen von dem tiefen Ton wohl zerrissen werden müssten.

XVI. Ich habe gesagt: die Verschiebung der Netzmembran

$$
B P=B B_{1} \cos \Varangle P B B_{1} \text {. }
$$

Es könnte also die Amplitude auch von $\Varangle P B B_{1}$ beeinflusst werden. Dieser Winkel ist jedoch in Wirklichkeit viel kleiner als in Fig. 1 angegeben. Denn in Wirklichkeit steht der innere Pfeiler ungefähr senkrecht, was auch aus den Figuren einiger Autoren wohl ersichtlich. Im Leben ist dies noch stärker der Fall, weil da die nach dem Lab. vestibulare hin gerichtete Spannung im inneren Pfeiler nicht verloren gegangen ist. Es differirt dann also $B P$ sehr wenig von $B B_{1}$. In der obigen Beschreibung ist überhaupt dieser Thatsache mit Absicht zu wenig Werth beigelegt worden, weil sonst diese erste Bewegungsform gar nicht denkbar wäre. Steht der innere Pfeiler senkrecht, so ist $\Varangle P B B_{1}$ so gross wie der von Retzius gemessene Winkel, den die Membrana reticularis mit der Basilarmembranebene macht. Er ist in der Basilarwindung $25^{\circ}$, in der Mittelwindung $35^{\circ}$, in der Spitzenwindung $23^{\circ}$. Bei dem Kaninchen variirt er etwas stärker.

XVII. Nach den Messungen von Retzius variiren die Grössen von Grundmembranbreite, innerem und äusserem Pfeiler und Abstande der Pfeilerfússe im selben Sinne wie nach den Hen sen'schen. Weil Retzius nicht die Extremen, sondern Punkte in der Basal-, der Mittel- und der Spitzenwindung in seiner Tabelle (Gehörorgan d. Wirbelthiere Bd. 2) erwähnt, springen die Unterschiede bei ihm nicht so deutlich, wenn auch deutlich genug, in's Auge. Die Figuren geben einen viel deutlicheren Eindruck der Verhältnisse, als Zahlen geben können. Die Fig. 3, 4 u. 5 sind unter 250 mal lin. Vergrösserung nach den Angaben in der Maasstabelle von Retzius construirt, Fig. 2 und 6 nach Hensen's Messungen (Zeitschr. f. wissschaftl. Zool. Bd. 13 oder Tonempf. S. 239). In Fig. 2 ist die Grösse von $A E$ etwas unsicher, weil nicht genau angegeben ist, welche von den Zahlen für die Grundmembranbreite bei den Zahlen der Pfeiler gehört; die Zuspitzung der Grundmembran proximalwärts ist bei einigen Thieren noch schärfer.

XVIII. Zweite denkbare Bewegungsform. Fig. 7 .

Es scheint nun aber die Spannung in den inneren Pfeilern oder die Kraft, mit der die von ihnen gebildete steife Leiste sich steil 
aufrecht zu stellen strebt (Helmholtz), eine ziemlich beträchtliche $\mathrm{zu}$ sein. Es muss daher die Grundmembran, wenn auch vielleicht in geringem Grade, eine Form annehmen, wie Fig. 7 zeigt. Es wird die Stelle der Membran, wo der Fuss des äusseren Pfeilers mit ihr verbunden ist, durch die Spannung im inneren Pfeiler mittelst des äusseren Pfeilers in die Höhe gezogen. Es wird die Spannung im inneren Pfeiler von derjenigen in der emporgezogenen Grundmembran im Gleichgewicht gehalten; ein solches Verhältniss von zwei sich entgegenwirkenden Spannungen iš ein für die genaue Uebertragung sehr gewünschtes Princip. Fs springt sofort in's Auge, dass durch die Verlaufsrichtung des äusseren Pfeilers die Spannung in der Zona pectinata $A \boldsymbol{E}$ viel grösser sein muss als in der Zona arcuata $C A$. Man betrachte hierfür besonders die nach den Messungen von Retzi us angefertigten Fig. 3-5, worin der schiefe Verlauf des äusseren Pfeilers viel stärker ausgeprägt ist. Die Fig. 1 und 7 sind ohne genaue Rücksicht auf Messungen gezeichnet worden.

XIX. Die grössere Spannung in der Zona pectinata (es gilt Folgendes auch für die dritte denkbare Bewegungsform) nun ist im schönsten Einklang mit der gerade in dieser Zone bestehenden deutlichen Querdifferenzirung und der viel grösseren Widerstandsfähigkeit in querer (= radialer) Richtung. Der verschiedenen Differenzirung der Zona pectinata und der Zona arcuata ist bis jetzt weder eine physiologische Bedeutung noch eine phylogenetische Erklärung beigelegt worden. Ich glaube, dass in der obigen Betrachtung sowohl die letztere wie die erstere enthalten ist. Das Entstehen des stärkeren Baues in. der Quere der Zona pectinata knüpft sich direct an die Differenzirung der zwischen den specifischen Haarzellen gelegenen Stützzellen und die diesen zukommende Function. Der schiefe Verlauf der Stützfasern findet sich schon beim Alligator sehr deutlich ausgeprägt; die Function des äusseren Pfeilers der Säuger ist nicht etwas ganz Neues, sie ist als eine Concentrirung der den schiefen Stützfasern der niederen Wirbelthiere obliegenden zu betrachten. Wenn auch bei den Reptilien keine inneren Pfeiler bestehen, so ist doch aus dem ganzen Bau des Corti'schen Apparates bei diesen Thieren ersichtlich, dass auch schon bei ihnen ein dem für den äusseren Pfeiler der Säuger beschriebenen analoger Zug auf die schiefen Statzfasern und mittelst dieser auf die Membrana basilaris ausgeübt wird.

XX. Nehmen wir bei der zweiten Bewegungsform wieder an, 
dass die die Bewegung der Grundmembran veranlassende Kraft sich über die ganze Breite derselben vertheilt. Wir müssen dann annehmen, dass die verschiedenen in der Lymphe suspendirten Theile keinen erheblichen Widerstand leisten und einfach mit himab- und hinaufbewegt werden; zugleich schreiben wir der Grundmembran eine gewisse elastische Dehnbarkeit zu. Bei der Abwärtsbewegung derselben werden ihre beiden Abschnitte $C A$ und $A E$ Gelegenheit haben, sich zu verkürzen, und das durch ihre Elasticität auch thun (siehe Fig. 7). Es entsteht dabei statt des Dreiecks $C A E$ ein Dreieck $C A_{1} E$, die Spannung im inneren Pfeiler wird vermehrt, weil dessen Kopf und mit ihm die Membr. reticularis nach aussen gezogen wird. Bei der Zugbewegung des Steigbügels würde das Umgekehrte stattfinden, die Grundmembran wird mit hinaufgedrängt und ihre Spannung vermehrt, der innere Pfeiler gehorcht seiner Tendenz und zieht die Membrana reticularis nach dem habium vestibulare hin. Die grösste Amplitude der Grundmembran findet sich bei dieser Bewegungsform also nicht in deren Mitte, sondern immer in der Anbeftestelle des Fusses des äusseren Pfeilers; es wird jetzt der grösste Ausschlag $A A_{1}$ (Fig. 7) mittelst des äusseren Pfeilers auf die Netzmembran und die Haarzellenhärchen übergebracht.

Es würden gerade bei dieser Bewegungsform der Membrana basilaris, bei welcher eine gewisse Elasticität derselben angenommen wird, die einzelnen Abschnitte eine Art von physiologischen Resonatoren vorstellen können, wie sie für eine Zerlegungstheorie wie die Helmholtz'sche doch eigentlich erforderlich wären. Denn die von verschiedenen Autoren gegen wirklich physische Resonatoren angeführten Erwägungen wiegen sehr schwer. Zur Erläuterung diene Folgendes.

XXI. Es ist aus den Helmholtz'schen Angaben nicht recht begreiflich, ob er sich eigentlich eine Massalbewegung oder eine Molecularschwingung vorgestellt hat als Ursache der Erregung der abgestimmten Schneckentheile und ob er sich diese wirklich als Resonatoren oder nur als Klangzerleger gewisser Art, m. a. W. als physische oder als physiologische Resonatoren, gedacht hat. Daraus, dass ẹr die physischen Gesetze des Mitschwingens, des Nachklingens und der Dämpfung auf sie anwendet, scheint hervorzugehen, dass er sie als physische Resonatoren betrachtet bätte. Dann brauchte jedoch die Molecularschwingung des Schalles nicht in Massenschwingung verwandelt zu werden, um dieselben zu erregen. Es scheinen die 
meisten Autoren es als die Meinung Helmholtz's aufgefasst zu haben, dass die Resonatoren des Ohres von Molecularschwingungen erregt werden. Es hat noch neulich Herr Pierre Bonnier in einer Arbeit, die er mir auf meine Bitte freundlichst zuschickte (De la Nature des Phénoménes auditifs, Réfutation de la Théorie de Helmholtz, Bull. scientif. de la France et de la Belgique, t. 25, 1895) es nöthig geurtheilt, das Stattfinden der Massalschwingung ausdrücklich hervorzuheben (S. 13-17), nachdem er zunächst die Unmöglichkeit des Mitschwingens irgend welcher Schneckentheile auf so tiefe Töne, wie wir sie wahrnehmen, betont hat (S. 9-11). - Aus einigen Aussagen $\mathrm{He}$ elm $\mathrm{mltz}$ 's scheint dagegen hervorzugehen, dass schon er eine Massalbewegung angenommen hat, auch als Ursache der Schwingung der Radialfasern der Grundmembran. Er sagt n. 1. (Tonempf. 5. Aufl. S. 240), dass die membranöse Querverbindung der Radialfasern „nur dazu dient, dem Drucke der Flussigkeit gegen diese Saiten eine Handhabe zu geben". Würde aber einfach eine Erregung von physischen Resonatoren (in casu von Saiten) durch Molecularschwingungen stattfinden, so brauchte dem Drucke der Flüssigkeit keine Handhabe ausser den anwesenden Saiten selbst gegeben zu werden. Dass er übrigens jedenfalls eine Massalbewegung als bestehend angenommen hat, gelit aus anderen Stellen hervor, so, wo er S. 241 sagt, dass obne eine Art Wellenbewegung in dem Wasser der Schneckentreppen sich die Membran gar nicht bewegen kamn; wo er S. 225 sagt, dass die Flüssigkeit nur ausweichen kann nach dem runden Fenster mit seiner nachgiebigen Membran, und wo er S. 222 etwas Aelnnliches hinschreibt und den Manometerversuch Politzer's erwälnt. -- Wir müssen mit Bestimmtheit eine vou den zwei Bewegungsarten als Ursache für die Erregung der Schneckentheile wählen; die Hörtheorie, die ich geben werde, ist das Resultat einer consequenten Durchführung des übrigens schon vor einem halben Jahrhundert von Ed. Weber angenommenen Princips der Massalbewegung. - Später hat Helmholtz in den Versuchen vol Hensen an den Gehörorganen der Crustaceen die Bestätigung der Möglichkeit des Bestehens von wirklichen Resonatoren gesehen. (Tonempf. S. 246 u. 247.)

XXII. Weil nun auch bei der zweiten denkbaren Bewegungsform der Grundmembran noch an eine Resonatorentheorie, wenn auch mit physiologischen Resonatoren und Vassalschwingung, gedacht werden könnte, werden wir auch hier die Amplitudenverhältnisse kurz besprechen.

E. Pfluger, Arclìv für Physiologie. Bd. i9. 
Was in $\S X V$ von dem Einfluss von den versehiedenen Werthen der Länge des inneren Pfeilers und des Pfeilerfussabstandes $(B C$ und $A C$ ) auf die Amplitude gesagt ist, das gilt hier genau ebenso. Das heisst ạlso, dass für einen bestimmten Werth der Amplitude $A A_{1}$ diejenige der Netzmembranverschiebung und der Härchen proximal ungefähr 4,5 mal so gross sein würde als distal. Die verschiedene Breite der Grundmembran könnten wir auf folgende Weise in Rechnung bringen bei der vorausgestellten Massalbewegung. Wird bei einer gewissen Hineinpressung des Steigbügels in einer Längseinheit des Schneckencanals ein bestimmtes Lymphequantum verschoben, so ist dieses proportional der Inhaltdifferenz der beiden Dreiecke $C A E$ und $C A_{1} E$ (s. Fig. 7), also prop. $A A_{1} \times C E$. Wo wir nun wissen, dass die Breite der Grundmembran $C E$ von proximal nach distal auf das Zwölffache wächst, da sehen wir, dass die Amplitude $A A_{1}$ distal, wo die tiefen Töne percipirt werden, bei einer bestimmten Lympheverschiebung durch die Längseinheit, hiernach eine $12 \mathrm{mal}$, und im Ganzen eine $4,5 \times 12=54$ mal kleinere sein würde als proximal, wo die hohen Töne mit ihren kleinen Amplituden ihr Gebiet haben. In dieser Hinsicht sind also die Maasse noch viel mehr ausgebeutet als bei der ersten denkbaren Bewegungsform. - Vergleichen wir nun einen Ton von $2^{12}$ mit einem solchen von $2^{5}$ Schwingungen. Es kann die Intensität jedes einzelnen Tons schon wechseln, dadurch allein muss die Amplitude der Härchen schon schwanken. Der Ton von $2^{5}$ würde jedoch bei derselben Intensität wie der von $2^{12}$ den Härchen, wenn die beschriebenen Einflüsse auf die Amplitude nicht bestünden, noch einen $128 \mathrm{mal}$ grösseren Ausschlag geben als der hohe Ton. Jetzt wird diese Zahl 128 aber auf $\frac{128}{54}$ reducirt. Dies ist also ein für die Sicherheit der Härchenzellen sehr willkommenes Ergebniss. Es fragt sich jedoch: wie es verhindert werden kann, dass die Intensität des tiefen Tons dabei sehr stark verkleint wird? Die Frage zwingt uns von selbst dazu, eine Theorie zu suchen, nach welcher die grössere Amplitude der tiefen Töne in irgend welchen anderen Verhältnissen ihren Ausdruck finde als in einem ebenso viel grösseren Ausschlag der Haarzellenhärchen.

XXIII. Dritte denkbare Bewegungsform. (Fig. 8.)

Ich habe in Cap. XVIII und XIX beschrieben, wie die Zona pectinata von den inneren Pfeilern mittelst der äusseren in Spannung gehalten wird, und wie darin die Erklärung der ganz vorwiegend 
der Zona pectinata zukommenden Gewebsdifferenzirung in der Quere enthalten ist. Es ist nun für mich geradezu überraschend, jetzt in der erwähnten "Réfutation de la Théorie de Helmholtz" von Bonnier Folgendes zu lesen (S. 10):

"Il n'était pas besoin d'invoquer les détails de sa" (= de la membrane basilaire) "structure, car les fameuses cordes de Nuel, qui ne sont que des épaississements dans le sens radial de la couche hyaline qui recouvrent la membrane, ue dépassent guère les piliers externes, et par conséquent ne peuvent être considérées comme tendues, n'ayant qu'une seule insertion fixe."

Es hat also Herr Bonnier auf einen Spanner für die Zona pectinata gefahndet, denselben jedoch nicht gefunden. - Die grössere Festigkeit der Grundmembran in radialer Richtung und die vermuthliche starke Spannung in dieser Richtung beschreibt Helmholtz auf S. 225 und 240 der "Tonempfindungen", die Spannung in den inneren Pfeilern (vgl. Cap. XVIII) auf S. 230. Dort hätte also Herr Bonnier die Spanner finden können, als welche ich ihm jetzt die inneren Pfeiler vorzustellen die Ehre habe. Diese greifen die Grundmembran durch die Vermittlung der äusseren Pfeiler gerade da an, wo Herr Bonnier es nöthig urtheilt, nämlich an dem letzteren Fusspunkte.

Wenn wir der Zona pectinata gern eine Spannung in radialer Richtung zuschreiben, so ist das nicht nur, wie einige Autoren zu denken scheinen, wegen der Streifung, die wir mit dem Auge wahrnehmen, sondern besonders wegen der leichten Zerreisslichkeit bei Spannung in der Längsrichtung gegenüber dem grösseren Widerstande in Richtung der Radialfasern.

Herr Bonnier gibt auf derselben Seite ein Citat nach P. Meyer, worin die Grundmembran sehr wenig elastisch genannt wird. Sie scheint das denn auch wirklich nicht zu sein; eine Dehnbarkeit, wie ich ihr die bei der zweiten denkbaren Bewegungsform zugemuthet babe, besitzt sie eigentlich nicht. Schon dieser Umstand würde mich nöthigen, noch nach einer anderen Bewegungsform mich umzusehen.

XXIV. Tieferes Eindringen in die anatomischen Verhältnisse der Schnecke zwingt uns, auf die ganz besondere Betheiligung der Zona pectinata bei der Grundmembranbewegung zu schliessen. Ist diese Wahl getroffen, so finden wir sie im schönsten Einklang mit folgenden Thatsachen, die an und für sich auch sehon zu demselben Schluss hinführen:

a) Während die ganze Basilarmembran von proximal nach distal zwölffach an Breite zunimmt, wächst die Breite der Zona pectinata 
auf das Zwanzigfache; wird also dem Zuwachs eine Bedeutung beigelegt, so wählen wir am liebsten die Zona pectinata.

b) Die mehrfach erwähnte Gewebsdifferenzirung in der Quere betrifft ganz vorwiegend die Zona pectinata.

c) Die Lage des Vas spirale gerade unter der Zona arcuata lässt eine geringere functionelle Wichtigkeit dieses Theiles als ein willkommenes Ergebniss erscheinen.

d) Das Resultat unserer Wahl ist, dass die Haarzellenhärchen, die an der Grenze unseres mikroskopischen Sehens liegen, im Allgemeinen sehr geringe Ausschläge bekommen, jedoch unter sehr genauer Uebertragung der Bewegung von der Grundmembran aus. Das Princip dieser Uebertragung ist das von mir in meinem vorigen Beitrag entwickelte Verschiebungsprincip. Dieses tritt noch reiner zu Tage, wo bei den sehr geringen Amplituden, welche die Härchen bekommen, und bei der ausschliesslichen Einwirkung der Lymphebewegung auf die Zona pectinata von einem Absteigen der Membrana tectoria nicht die Rede zu sein braucht.

e) Einige Formationen in der Schnecke, denen noch keine Berleutung beigelegt war, werden sich fortan einer solchen erfreuen können.

Bei den beiden schon beschriebenen Bewegungsformen haben wir angenommen, dass die Kraft, von welcher Art sie denn auch sein möchte, ungefähr gleichmässig die ganze Breite der Grundmembran angreift. Daher müssten wir, wenigstens für eine massale Bewegung der Lymphe, die ganze Corti'sche Papille und die Membrana tectoria nit hinab- und hinaufsteigen lassen, wie schon in Cap. II hervorgehoben.

XXV. Ich stehe von jetzt an unersehütterlich fest auf dem Standpunkt, dass nur der massalen Lympheverschiebung eine Rolle bei der Erregung der Schneckenapparate zukommt, und es fällt mir jetzt noch eine Stelle der "Tonempfindungen" ein, woraus auf's Neue hervorgeht, dass auch Helmholtz ursprunglich nur an die Massalbewegung gedacht hat für die Erregung der Apparate. Er hebt da hervor, wie für ein schnelles Hin- und Hergetriebenwerden des Labyrinthwassers, "wie es bei den Schalloscillationengeschieht", viel erheblichere Druckkräfte nöthig sind als für die Luft des Gehörgangs. Wo er dann auch etwas weiter sagt, dass die mechanische A ufgabe des Trommelhöhlenapparats ist, eine Bewegung von grosser Amplitude und geringer Kraft, welche das Trommelfell trifft, zu verwandeln 
in eine von geringer Amplitude und grösserer Kraft, die dem Labyrinthwasser mitzutheilen ist, da möchte ich lieber Folgendes sagen:

Die mechanische A ufgabe des Trommelhöhlenapparats ist, die Molecularbewegung der Luft im äusseren Gehörgang zu verwandeln in eine Massalbewegung des Labyrinthwassers. Die Amplitudenverkleinerung und Kraftvergrösseruug erscheinen dann als nothwendige Mittel, um dieses Ziel zu erreichen. - Innerhalb des Gehörorgans kann nicht mehr von Schall die Rede sein. Das Trommelfell bildet die Grenze zwischen Schall und Nichtschall. Das Gehörorgan (und um so weniger die Schnecke) dient nicht, um den Schall zu resonniren, sondern um denselben zu tödten, das heisst in Energie einer auderen Sorte umzusetzen. Die Schallenergie wird nicht unmittelbar, sondern längs des $Z$ wischengliedes der mechanischen und hydraulischen Massalbewegung des Trommelböhlenapparats, des Labyrinthwassers und des Corti'schen Apparats in Nervenenergie übergeführt.

XXVI. Bei der dritten denkbaren Bewegungsform der Grundmembran nehmen wir nur dasjenige an, was Thatsache ist:

1. Die Spannung im inneren Pfeiler, die mittelst des äusseren Pfeilers auf die Membrana basilaris übergebracht, und ganz vorwiegend von derjenigen in der Zona pectinata im Gleichgewicht gehalten wird.

2. Die Undehnbarkeit der Grundmembran für die auf sie einwirkenden Kräfte und für die Art, worauf diese bei der zu beschreibenden Bewegungsform dieselbe sich zu bewegen veranlassen.

Durch den spiraligen Verlauf des Schneckencanals wird bei der Lympheverschiebung, die der Hineinpressung der Steigbügelplatte folgt, immer eine besondere Staung entstehen nach Aussen (in der Richtung der beiden Pfeile links von der Membrana Reissneri Fig. 8), weil die Richtung der Flüssigkeitsbewegung möglichst wenig zu wechseln versucht. Danach wird die Membrana Reissneri die in Fig. 8 angegebene Ausbuchtung bekommen (bei jeder Stossbewegung des Steigbügels). Die Lympheverschiebung wird nun hauptsächlich stattfinden in der Richtung des grossen Pfeiles. Fs bilden hier die Hensen'schen Stützzellen einerseits, die Stria vascularis mit dem Vas prominens (das in der Zeichnung noch nicht einmal prominirt) andererseits, den Eingang zu einer Rinne, dessen Boden die Zona 
pectinata ist. Wer zweifelt jetzt noch, dass diese die Hauptrolle spielt bei der Bewegung der Grundmembran ? ${ }^{1}$ ). An den Zeichnungen der verschiedensten Autoren (vgl. bes. Retzius Gehörorg. d. Wirbelth. Bd. 2 oder Gegenbaur Anatomie Bd. 2 S. 574; 5. Aufl.) sind diese Verhältnisse mit der grössten Deutlichkeit zu sehen: man richtet auf der Mitte (oder eigentlich etwas mehr nach oben) der Membrana Reissneri eine senkrechte Linie auf, diese führt unmittelbar nach der Zona pectinata hin. Dieser Function ist die Membrana Reissneri gewachsen, solange sie nur nicht zerreisst bei der Schallwahrnehmung. Dass sie das nicht thut, wissen wir. Die Function, die ich der Stria vascularis und den Hensen'schen Stützzellen beilege, ist so einfach, dass sie eine Erläuterung nicht braucht. Man könnte für die Leistung der letzteren noch hinzufügen, dass sie eine directe Einwirkung auf die Haarzellen verhindern. Es ist ganz deutlich, dass die Membrana tectoria und die unter ihr liegenden Gebilde nur einer sehr geringen hinabdrückenden Kraft Widerstand $\mathrm{zu}$ leisten haben bei der Lympheverschiebung. Wir können jedoch noch Folgendes hinzufingen. Die Deckmembran ist nicht nur eine weiche Masse, sie besitzt an der Unterseite einen härteren Ueberzug. und an ihrer äusseren Grenze einen festeren Ring, der mit dem härteren Ueberzug ein Ganzes bildet. Stellen wir uns jetzt die Deckmembran in ihrer spiraligen Ausbreitung vor, so können wir nur sagen, dass sie im Stande ist und daraufhin gebaut ist, der Lympheverschiebung einen nicht zu vernachlässigenden Widerstand zu bieten. Die $\mathrm{Z}$ on a pectinata jedoch kann ausweichen, und zwar ohne dass ihr dafür eine Dehnung zugemuthet wird: nur hat sie die Spannung im inneren Pfeiler zu überwinden.

XXVII. Die Fig. 8 gibt nun ferner den Ruhestand der Zona pectinata und des Pfeilerphalangenapparats und daneben den $\mathrm{Zu}$ stand einer übertriebenen Ausbuchtung. Eine Ausbuchtung findet statt bei jeder Hineinpressung des Steigbügels. Dadurch wird der Fusspunkt des äusseren Pfeilers $A$ (Fig. 8) dem Lig. spir. etwas genähert, n. l. um den Abstand der punktirten Linien $a$ und $b$. Nennen wir die Tiefe der Ausbuchtung $S$ und die Länge von $A E l$, so wissen wir, dass $a b=\frac{8}{3} \times \frac{S^{2}}{l}$ ist (für die kleinen in Betracht kommenden

1) Wollte man der Membrana Reissneri keine Function beilegen, so kame man dennoch durch die Lage der Zona pectinata in Verband mit dem spiraligen Verlauf des Schneckencanals zu demselben Schluss. 
Ausbuchtungen). $A A_{1}$ darf in diesem Fall als gerade Linie betrachtet werden und ist also proportional $a b$. Es ist also auch $B B_{1}$ (die Grösse der Netzmembranverschiebung bei geringen Ausbuchtungen) proportional $a b$. Aus dem Werth $\frac{8}{3} \times \frac{S^{2}}{7}$ geht hervor, dass die Netzmembranverschiebung und also die Amplitude der Haarzellenbärchen immer eine sehr geringe ist. Die Vergrösserung der Kraft, die dabei auftritt, wird benutzt, um die Spannung im inneren Pfeiler und weiter den Widerstand der (spiralig vervollständigt gedachten) Membrana reticularis zu überwinden. Es fällt hierbei selbstverständlich einem Jeden die Uebereinstimmung mit der von $\mathrm{Helm}$ holtz beschriebenen Bewegung des Hammerstiels auf.

Dass wahrscheinlich die äussere Hälfte der Zona pectinata etwas mehr ausgebuchtet wird als die innere, ist nur im Einklang mit der deutlicheren Querstreifung in der Nähe des Lig. spirale.

Es ist in Beziehung auf diese dritte Bewegungsform der Grundmembran noch zu bemerken, dass für die breiteren Strecken derselben eine genaue Uebertragung eben nur bei dieser Form der Bewegung möglich erscheint.

XXVIII. Stoss - und Zugbewegung.

Ich habe fast immer nur gesprochen von dem, was bei der Stossbewegung des Steigbügels geschieht. Fs ist jetzt die Frage zu beantworten, was die Zugbewegung desselben für Veränderungen nach sich zieht. Wenn der Steigbügel hineingepresst worden ist, so ist die Zona pectinata irgendwo nach unten ausgebuchtet, wie aus Fig. 8 ersichtlich. Wird jetzt der Steigbügel herausgezogen, so wird zunächst die Ausbuchtung sich wieder ausgleichen, bis dieselbe unter dem Einfluss der Spannung im inneren Pfeiler ihre gestreckte Lage wieder bekommen hat. Jetzt kommen die negativen Ordinaten der Curve, jetzt erst könnten wir, von der Gleichgewichtslage aus rechnend, von einem eigentlichen Herausgezogenwerden des Steigbügels reden. Nehmen wir an, dass eine solche, also eine Zugbewegung des Steigbügels, wirklich stattfände, so würden die Factoren, die wir besprochen haben, gar nicht bestehen. Denn es würde das Andrängen der Lymphe von unten her, von der Scala tympani aus stattfinden; in dieser Scala besteht jedoch weder eine Membrana Reissneri, noch eine Deckmembran, auch liesse sich der Einfluss des spiraligen Verlaufs des Scbneckencanals nicht geltend machen. Es würde von der Fenestra rotunda aus die Lymphe nach oben gestaut werden, zunächst gegen die schmalste und unnachoiebigste Gegend der 
Grundmembran, da, wo dieselbe sich fast scharf zuspitzt. Es würde eher eine Begünstigung der Zona arcuata als der Zona pectinata stattfinden. Es würde nur die ganze Grundmembran etwas gedebnt werden können, wobei der Fuss des äusseren Pfeilers etwas gehoben würde, die Membrana reticularis etwas nach innen, also in umgekehrter Richtung als bei der Stossbewegung des Stejgbügels, verschoben würde. Bei der Dehnung der Grundmembran würde aber sogleich eine ungeheure Spannung in derselben auftreten, es würde also die Lympheverschiebung und auch die ganze Zugbewegung des Steighügels eine beträchtliche Hemmung erfahren. Wir merken also einen grossen Unterschied zwischen dem Mechanismus bei der Stosshewegung und der Zugbewegung. Es ist dieses Ergebniss ein willkommenes, wie aus weiteren Erwägungen hervorgehen wird. Würde die Zona pectinata bei der Zugbewegung nach oben ausgebuchtet werden, so würde wierler in Folge des Zuges nach dem Lig. spirale hin, eine Senkung des Fusses des äusseren Pfeilers und somit eine Verschiebung der Membrana reticularis nach Aussen hin erfolgen. Es würden also die Haarzellen bei der Zugbewegung ein ähnliche Erregung wie bei der Stossbewegung erfahren. Dies werden noch weitere Erwägungen sehr unwahrscheinlich erscheinen lassen.

XXIX. Wir finden nun aber diese Beeinträchtigung der Zugbewegung nicht nur im Corti'schen Apparat selbst. Denn wie Hensen nachgewiesen hat, findef die Schwingung des Trommelfells nicht um ihre Ruhelage, sondern um einen etwas einwärts davon gelegenen Punkt statt. Es scheint hier also schon eine directe Hemmung der Zugbewegung stattzufinden. Es könnte dies daher stammen (und das wird auch wohl so sein), dass die Gebilde, deren Spannung bei der Zugbewegung erhöht wird (z. B. Lig. mallei externum und superius), weniger dehnbar sind als diejenigen, deren Spannung bei der Stossbewegung eine Vermehrung erfährt (z. B. die Ringfasern des Trommelfells). Auch könnte man sich vorstellen, dass der Tonus des Muse. Tensor tympani bei jeder Zugbewegung erhöht würde; dafür sind aber die Schalloscillationen wahrscheinlich wohl zu schnell. - Es ist jerhch überhaupt die Frage, ob je eine Zugbewegung des Steigbügels aus der Ruhelage zu Stande kommt. Denn wir wissen ja, dass das Sperrgelenk zwischen Hammer und Amboss derart ist, dass der Amboss, wenn das Trommelfell mit dem Hammer nach Aussen gezogen wird, gar nicht mitzugehen braucht: die Sperrzähne der Gelenklächen weichen dann von einander, sie 
„packen" nicht. Diesen Thatsachen ist noch hinzuzufügen, dass ein Blick auf den Durchschnitt des Foramen ovale mit dem Steigbügel in Hensen's Physiologie des Gehörorgans uns den Eindruck gibt, dass eine Zugbewegung des Steigbügels im Leben eigentlich absolut nicht zu Stande kommen könnte. Ich weiss nicht, ob diese Verhältnisse bẹi den verschiedenen Manometerversuchen berücksichtigt worden sind. Helmholtz selbst hat der Einrichtung des Hammerambossgelenks zwei Bedeutungen beigelegt, erstens, dass der Steigbingel nicht aus dem ovalen Feuster ausgerissen werden kann, wenn die Luft im Gehörgang erheblich verrünnt wird, zweitens, dass bei starken tiefen Tönen und gewissen Zusammenklängen ein Klirren entstünde, das im letzteren Fall vielleicht eine Rolle bei dem $\mathrm{Zu}$ standekommen des Differenztones spielen könnte. Ich glaube, dass dieses Klirren nur mit grosser Vorsicht in den Kreis der Betrachtung zu ziehen ist. Es ist die Frage, ob nicht gerade beim angestrengten Hören nach starken Tönen und Zusammenklängen (und zwar um Combinationstöne herauszubören) das Klirren in den Ohren des grossen Meisters vielleicht einem unbewussten (oder reflectorischen) Innerviren des Musc. Tensor Tympani (resp. auch M. stapedius) zugeschrieben werden muss. Ich kann den Tensor sehr leicht isolirt innerviren, un£efähr 15 Secunden lańg, und bei festem Schliessen der Augen ungefähr 45 Secunden. Bei mässiger Innervation tritt ein exquisites Muskelgeräusch, bei starker ein Klirren daneben ein.

XXX. Kommen wir auf die Zugbewegung zuruck, so geben alle die in Cap. XXVIII und XXIX betonten Verhältnisse mir den Eindruck, dass bein Bau des Ohres nur der Stossbewegung und gar nicht der Zugbewegung, insoweit diese durch wirklich negative Ordinaten in der Curve der Steigbügelbewegung vorgestellt werden könnte, Rechnung getragen worden ist. Bei der Stossbewegung: werden ganz besondere Apparate in Spannung versetzt (d. h. in grössere Spannung); im Trommelfell die Ringfasern, über deren eigenthümliche Leistung man Tonempf. 5. Auf. S. 220 nachlese (man wird dann zugleich die überraschende Uebereinstimmung der Mechanik des Trommelfells und der von mir Cap. XXVII beschriebenen Wirkung der Zona pectinata bemerken); in Corti'schen Apparat die starren inneren Pfeiler. Die Zurückbewegung des Hammerstiels geschieht gewissermaassen passiv durch die gespannten Ringfasern, die Zurückverschiebung der Membrana reticularis ebenso durch die vermehrte Spannung im inneren Pfeiler. Diese Zurúckschiebung der Netzmembran könnte gar nicht activ von einer Hinaufbewegung der 
Grundmembran bewerkstelligt werden, weil der äussere Pfeiler kein Pfeiler, sondern ein biegsamer Faden ist. Weil wir nun wohl annehmen müssen, dass die Erregung der Haarzellen, analog der Innervation der Muskeln, aus getrennten Einzelerregungen entsteht, so stehe ich nicht an, nur entweder der Stossbewegung oder der Zugbewegung, und zwar in Verband mit obigen Betrachtungen nur der Stossbewegung eine Rolle bei der Erregung der Haarzellen zuzumuthen. Vermuthen können wir dann ferner, dass bei der Stossbewegung (und Auswärtsbewegung der Membrana reticularis) der in den Haarzellen aufgefundene spiralige Faden angezogen, bei der Zugbewegung (Einwärtsverschiebung der Netzmembran) relascirt werde; bei dem Anziehen fände die Erregung statt.

Es ist die Sache nun nicht so aufzufassen, als ob nur die positiven Curvenordinaten etwas mit der Perception auszustehen hätten, die negativen aber gar nichts; wir müssen vielmehr unterscheiden zwischen Stossbewegung im Sinne von "Ansteigen der Curve (= Positiverwerden der Ordinaten)" und Zugbewegung im Sinne von "Abfallen der Curve (= Negativerwerden der Ordinaten) “. Die erwähnte Hemmung der Zugbewegung bewirkt nicht so sehr ein Wegfallen aller negativen Ordinaten als ein Hinabrücken der Gleichgewichtslinie der Curve in negativer Richtung. Dieses Princip würde eine weitere Betrachtung verdienen.

XXXI. Dass für das Ohr Stoss- und Zugbewegung nicht identisch sind, hat aus Versuchen über Schwebungen von unreinen Consonanzen, also aus ganz anderen Gründen als ich, schon Sir William Thomson (二 Lord Kelvin) 1878 vermuthet, wo er sagt (On Beats of Imperfect Harmonies; Proc. Royal Society of Edinburgh, Session 1877-1878 S. 610):

"Whatever may be the physiological theory by which the beats are to be explained, it is an interesting fact, that the ear does distinguish, as it were, between push and pull on the tympanum..."

Er scheint auch das grössere Gewicht zu legen auf das Ansteigen und Abfallen der Curve; dies geht aus folgenden Worten hervor (S. 609).

"In the one case the pressure of air close to the ear rises very suddenly to, and falls very suddenly from, its maximum and there is a comparatively slom variation of pressure for a few ten thousandths of a second on each side of the instant of minimum pressure; in the opposite phase-relation there is a slow change before and after the time of maximum pressure, and a rapid change before and 
after the time of minimum pressure." Es betrifft dies den Unterschied zwischen zwei übrigens gleichen Curven, davon jedoch die eine die Ordinatenumkehrung der anderen darstellt, die eine scharfe Gipfel oben, die andere unten besitzt. Es scheint Lord Kelvin zu meinen, dass sowohl der ansteigende wie der absteigende Theil der Curve eine Erregung gibt, jedoch jeder eine verschiedenartige. Uebrigens betrifft es bei ihm die Superpositionscurve, ohne Rücksicht auf die Zerlegung. Es würde mich zu weit führen, jetzt die interessante Frage des Einflusses des Phasenunterschiedes auf die Klangfarbe mit den beschriebenen Verhältnissen in Verband zu bringen.

Herr Pierre Bonnier hat die ausschliessliche Erregung während des positiven Theils der Curve vermuthet. Er sagt (Société de Biclogie, Séance du 23 Février 1895): „L'irritation cellulaire ne dure done qu'une demi-phase, e'est-à-dire qu'elle se produit pendant la période positive de la phase ondulatoire." Man könnte denken, dass er mit "période positive" den ansteigenden Theil der Curve meinte und also das Gleiche sagte wie ich. Dass er jedoch wirklich dasjenige meint, was er sagt, scheint aus den höher oben gebrauchten Worten: "... en considérant comme positives les positions inférieures au niveau d'équilibre, et comme négatives les supérieures" hervorzugehen, die die Grundmembranbewegung betreffen. Auch beschreibt er, wie nur beim Absteigen der Corti'schen Papille aus der Gleichgewichtslage das die Erregung bewirkende Tiraillement statt hat. Dieses Tiraillement ist das Umgekehrte des Hensen-Helmholtz'schen Anstossens gegen die Corti'sche Membran (vgl. Cap. I); nur ist nicht recht deutlich, wie die Bewegung der Corti'schen Membran nach unten, durch die Befestigung auf den Huschke'schen Zähnen gehemmt wird.

XXXII. Princip einer neuen Hörtheorie.

Betrachten wir die Fig. 9, einen zur Membrana basilaris senkrechten Längsschnitt durch die ausgerollte Schnecke.

Was geschieht, wenn die Steigbügelplatte in das Foramen ovale hineingetrieben wird? Es wird dann ein Quantum Lymphe von der Fenestra ovalis (resp. dem Eingang in die Scala vestibuli vom Vestibulum her) nach der Fenestra rotunda verschoben, weil diese die einzige nachgiebige Stelle ist. Denken wir die Membrana basilaris fort, so wurde die Lympheverschiebung so unmittelbar wie nur möglich stattfinden. Eine merkbare Lymphebewegung würde also nur im proximalsten Theile der Schnecke zu verspüren sein. Es 
findet sich nun aber zwischen den zwei Fenstern und zwischen den beiden, diesen entsprechenden Scalae die mehr oder weniger nachgiebige Zona pectinata. Ein Blick auf die Fig. 2-6 genügt nun, um zu sehen, dass das Mehr der Nachgiebigkeit für ein bestimmtes Lymphequantum in die distale Schneckengegend (Fig. 6), das Weniger in die proximale (Fig. 2) fällt. In der proximalsten Gegend ist die Breite der Zona pectinata, besonders auch gegenüber der Grösse des inneren Pfeilers, äusserst gering, so dass ein gewisses Lymphquantum hier nur schwer Platz findet. Dies wird um so besser, je mehr wir in die distalen Gegenden gerathen, und Fig. 6 lässt in Beziehung auf die Leichtigkeit, womit das gleiche Lymphequantum hier eine genügende Ausbuchtung der Zona pectinata bewerkstelligen wird. um Platz zu finden, gar keinen Zweifel übrig. - Was wird also geschehen? - Sobald die Steigbügelplatte hineingepresst wird, entsteht eine ganz geringe Ausbuehtung bei $a$ (Fig. 9). - Diese Ausbuchtung wird aber sogleich bei ihrem Entstehen in Folge der aufgeweckten Spannung distalwärts fortwandern, indem da das gleiche Lymphequantum leichter Platz findet. Eine Ausbuchtung muss irgendwo fortbestehen, weil der Raum oberhalb der Grundmembran verkleinert bleibt und die Lymphe sich weder zusammendrücken lässt, noch irgendwo entweichen kann ausser nach unten, wo durch die Ausbuchtung des Tympanum secundarium Platz gemacht wird. Es sei sogleich bemerkt, dass ein bestinmtes Lymphequantum seine Ausbuchtung ruhig längs der Zona pectinata sich fortpflanzen lassen kann; es braucht dafur licht eine grössere Lymphesäule verschoben zu werden, je grösser der Abstand vom Foramen roturılum wird; demn wo proximalwärts die Ausbuchtung versehwindet, da wird Platz gemacht für das in der nächsten distalwärts gelegenen Strecke weggedrückte Lymphequantum. Dies gilt nicht, solange der Steigbügel noch weiter vordringt; dann besteht jedoch auch diese grosse Triebkraft, die das Tympanum secundarium weiter ausbuchtet. Solange diese weitere Hineinpressung stattfindet, also während des ansteigenden Theiles der Curve ( $A B C$ Fig. 10), entsteht bei $a$ die geringe Ausbuchtung fortwährend auf's Neue.

XXXIII. Hat die Curve ihren Höhepunkt erreicht ( $C$ Fig. 10), so fängt die Steigbügelplatte sich zurúckzuziehen an, es entstehen bei $a$ keine neuen Ausbuchtungen mehr; die alten, davon sich die am ersten (im Zeitpunkt $A$ der Curve $A B C D E$ Fig. 10) gebildeten jetzt bis $c$ (Fig. 9) fortgepflanzt haben, setzen ihre Wanderung in distaler Richtung fort, sie bilden zusammen eine Welle. Während diese sich aber in der 
Zeitstrecke zwischen $C$ und $E$ (Fig. 10) fortpflanzt, wird sie eben durch das Herausgezogenwerden der Steigbügelplatte an ihrem proximalen Ende stetig verkürzt, d. h. dass die Ausbuchtung der Zona pectinata am proximalen Theile der Welle allmälig versehwindet. Dies findet 1. I. leichter statt, als dass die Grundmembran etwa bei a nach oben eine Ausbuchtung erfahren sollte (vgl. Cap. XXVIII). Dieses Verschwinden der Ausbuchtung von proximal nach distal findet zugleich mit dem Fortwandern der ganzen Ausbuchtungswelle in distaler Richtung statt. Wenn die im Zeitpunkt $A$ (Fig. 10) gebildeten Aushuchtungen, mit andern Worten, weun das distale Ende der Welle bis $d$ (Fig. 9) fortgeschritten ist (d. h. also im Zeitpunkt $D$ der Curve $A B C D E$ oder nach ${ }^{3 / 4}$ der ganzen reriode), ist die ganze Welle viel kürzer als im Zeitpunkt $C$, d. h. am Ende der halbeu Periode, wo die Welle die Länge $a c$ (Fig. 9) hatte. Im Zeitpunkt $E$, d. h. am Ende der ganzen Periode, ist das distale Ende der Welle in $e$ (Fir. 9) angelangt, zugleich ist jetzt die ganze Welle verschwunden, weil die Steigbùgelplatte gänzlich zurückgezogen ist; die Zona pectinata ist wieder gestreckt. Das Allmälirgschwinden der Ausbuchtung von proximal nach distal in der zweiten Hälfte der Periode verändert nichts an der Thatsache, dass iil dieser zweiten Hälfte nach einander alle Punkte zwischen $c$ und $e$ (Fig. 9) eine Ausbuchtung erfahren, erregt werlen, während in der ersten Mälfte alle Punkte zwischen $a$ und $c$ und nur diese betheiligt werden. Alle Punkte zwischen $a$ und $e$ werden im Zeitabschnitt einer ganzen Periode nach einander von dem Beginn der Erregung betroffen. Es sei sogleich nebenbei bemerkt, dass ein Ton von doppelter Schwingungszahl, also die Octave des besehriebenen, nie andere Punkte erregen wird als die zwischen $a$ und $c$ gelegenen, d. h. als die Punkte, die in der ersten Hälfte der Periode des Grundtons ihre erste Erregung erfahren, und dass in der zweiten Hälfte der Grundtonperiode, wo die zwisehen $c$ und $e$ (Fig. 9) gelegenen Punkte ihre erste Erregung bekommen, die Octave auf's Neue die zwischen $a$ und $c$ gelegenen eine Erregung erleiden lassen wird. Es hat auf diese Thatsache keinen Einfluss, wenn vielleicht die Fortpflanzungsgeschwindigkeit der Ausbuchtungen nicht, wie in Fig. 9 vorgestellt, eine gleichmässige, sondern eine regelmässig abnehmende sein würde.

Der Punkt $e$, bis zu welchem die Ausbuchtungen sich uberhaupt fortpflanzen können, wird nur bestimmt von der Zeit, die vorhanden ist, für die distale Fortpfianzung, d. h. von der Periode des Tons. Ist die Schwingungszahl des Tons $n$, so rflanzen sich die Aus- 
buchtungen einmal in jeder Periode, d. h. einmal in jeder $\frac{1}{n}$ Secunde, bis $e$ fort. Ist der Ton von vier Mal so grosser Schwingungszahl, also zwei Octaven höher, so ist seine Periode $\frac{1}{4 n}$, die Ausbuchtungen werden sich in diesem Fall nur bis zum Punkt $b$ fortpflanzen; denn wenn sie bis zu diesem Punkt fortgeschritten sind, besteht eben keine Ausbuchtung mehr, weil der Steigbügel sich bis auf seinen Ausgangspunkt zurückgezogen hat.

XXXIV. Wo ich von nach einander bei $a$ entstehenden Ausbuchtungen geredet habe, da ist das nur der Deutlichkeit wegen; die Steigbügelplatte dringt, solange die Curve ansteigt, continuirlich weiter vor; von der dadurch entstehenden Ausbuchtung fliesst fortwährend ein Theil distalwärts ab. Die Bildung der Ausbuchtungen am Anfangstheile der Grundmembran entspricht dem Zeitverlauf $A$ bis $C$ (d. h. dem Theil der Abscisse zwischen den Projectionen von $A$ und $C$ ), Fig. 10 Curve $A B C D E$. In Zeitpunkt $B$ wird die grösste Ausbuchtung gebildet (das grösste Quantum Lymphe pro Zeiteinheit zwischen Steigbügelplatte und Foramen rotundum verschoben), weil hier die Curve am steilsten ist. Fliessen nun die Ausbuchtungen $a b$, so werden immer die im Zeitpunkt $B$ gebildeten Ausbuchtungen den Gipfel darstellen in der Welle der in der Zeitstrecke zwischen $A$ und $C$ gebildeten Ausbuchtungen. Im Zeitpunkt $C$ sind sämmtliche Ausbuchtungen gebildet, von jetzt an fliessen sie, eine Welle formend, distalwärts $a b$, unter fortwährender proximaler Verkürzung dieser Welle, bis der äusserste von der Periode gestattete Punkt und zugleich der Punkt des Todes der Welle erreicht worden ist. Es wird also ein einfacher Ton in jeder Periode eine bestimmte Strecke (die immer am Foramen rotundum anfängt) der Corti'schen Papille, in casu die Strecke a $e$ (Fig. 9), auf eine bestimmte regelmässige Weise, einmal erregen. Ein höherer Ton wird einen Theil (lieser Strecke (der immer vom Foramen rotundum ab gemessen wird), auf die gleiche regelmässige Weise, einmal in seiner eigenen Periode, also öfters in jeder Periode des tiefen Tons, erregen. Werden der tiefe und der hohe Ton, z. B. $A B C D E$ und $A, B_{\prime \prime} C_{\prime \prime} D_{, \prime} E_{\prime \prime}$ von Fig. 10, zugleich gehört, so wird vom tiefen Ton zwar die gleiche Strecke wie zuvor erregt, jetzt aber nicht in der gewöhnlichen regelmässigen Weise, d. h. nicht mit der gewöhnlicben Vertheilung der Intensität der Erregung über die ihm eigenthümliche Strecke. Ein gewisser Theil, am Foramen rotundum gelegen, wird in der Periode 
des höheren Tons grössere Ausbuchtungen und stärkere Erregungen bekommen. Dadurch besteht zwar der tiefe Ton fort, er bekommt jedoch einen gewissen, von der einfachsten Erregung abweichenden Charakter, d. h. er bekommt eine $\mathrm{Klang}$ a r be. Zugleich ist aber die kleine an Foramen rotundum gelegene Strecke, die dem hohen Tone eigenthümlich ist, genügend markirt durch die Fortpflanzung der tieferen Ausbuchtung und dadurch grösseren Erregung der Haarzellen gerade über diese ihm eigene Strecke. Neben dem tiefen Ton kann also, wenn auch auf diese Markirung die Aufmerksamkeit gelenkt wird, der hohe Ton gehört werden.

Eine einfachere Erklärung kann man wohl nicht verlangen von der Thatsache: dass wir eine Klangmasse, die aus einem Grundton mit einer Anzahl von Obertönen besteht, davon einige selbst dem Grundton an Intensität gleichkommen oder ibn selbst übertreffen können, wahrnehmen als den Grundton, nur mit anderer Klangfarbe. Dies ist nach einer Resonatorentheorie, wo tiefe und hohe Töne absolut von einander getrennt werden, durchaus unbegreiflich. Es ist nicht weniger deutlich, dass, wenn ein gewisser hoher Ton stark vergegenwärtigt ist, seine Strecke besonders stark markirt wird und er deutlicher in den Vordergrund treten wird als der schwache tiefe Ton.

XXXV. Vergleichen wir die Curven $A B C D E$ und $A, B_{, \prime}, D, E$, von Fig. 10, so sehen wir, dass ihre physikalische Intensität die gleiche ist. Denn diejenige des ersten (tiefen) Tons ist prop. $n^{2} A^{2}$, die des letzteren (hohen) Tones prop. $64 n^{2} \times \frac{A^{2}}{64}$, weil die Periode, sowohl wie die Amplitude des tiefen acht Mal grösser sind als die des hohen Tons. Man könnte sich nun fälschlich vorstellen, dass der tiefe Ton mit seiner ungeheuren Amplitude kolossal viel tiefere Ausbuchtungen geben würde als der hohe Ton von derselben physikalischen Intensität. Man behalte jetzt aber in Auge, dass die Ausbuchtungen durchaus nicht an Tiefe zunehmen mit dem Zunehmen der Curvenamplitude zwischen den Zeitpunkten $A$ und $C$, weil ja die bei $a$ Fig. 9 gebildeten Ausbuchtungen sogleich distalwärts abfliessen; dass dieselben nur von $A$ bis $B$ etwas an Tiefe zunehmen durch das Steilerwerden der Curve, so dass sie von $B$ bis $C$ wieder eben so stark abnehmen. Die Steilheit nimmt bei Tönen von gleicher Amplitude wie $A B, C$, und $A, B, C, D_{n}, E_{n}$ mit der Schwingungszahl zu; bei Tönen von gleicher Schwingungszahl, wie $A B C D E$ und $A B, C$, (nur für die Hälfte gezeichnet) mit der Amplitude. Aus den Figuren ist denn auch ersichtlich, dass 


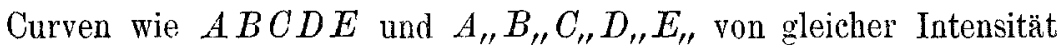
auch überall die gleiche Steile haben. Die Ausbuchtungen, die der Ton $A B C D E$ am Anfang der Schnecke bildet, sind also nicht tiefer als die des Tones $A_{n,} B_{, \prime} C_{n} D_{n,} E_{\prime \prime}$.

Bei dem Ton $A B C D E$ pflanzen sich die Ausbuchtungen acht Mal so weit fort, bei dem Ton $A_{n} B_{n} C_{n} D_{n}, E$, pflanzen sie sich über eine acht Mal kleinere Strecke acht Mal öfter fort.

Hieraus ergibt sich folgendes interessante Resultat:

Von zwei Tönen gleicher physikalischer Intensität, aber verschiedener Tonhöhe, kommt die Intensität, die der tiefe Ton der ihm als solehem inhärenten grossen Amplitude verdankt, zum Ausdruck in der grossen Längsstrecke der Corti'schen Papille (also der grossen Anzahl der Haarzellen), die erregt wird; während die Intensität, die der hohe Ton der ihm eigenthümlichen grossen Schwingungszahl verdankt (und die ihm sonst wegen der kleinen Amplitude fehlen würde), zum Ausdruck kommt in der häufigen Erregung der kleinen von ihm befallenen Strecke der Corti'schen Papille (d. h. der häufigen Erregung einer kleinen Auzahl von Haarzellen). Die Amplitude, welche die Haarzellenhärchen erhalten, hat für eine bestimmte Intensität einen bestimmten Werth, unabhängig von der Tonhöhe. Bei Tönen verschiedener Intensität und Tonhöhe richtet sich dieser Werth nach den Intensitäten, ohne Rücksicht auf die physikalischen Amplituden der Töne (vgl. das Postulat am Schluss des Cap. XXII).

Es könnte dies vielleicht einmal den Weg zeigen nach den Beziehungen zwischen der physiologischen und physischen Intensität.

XXXVI Ich habe in Cap. XXXIV schon unwillkürlich angenommen, dass die Tonhöhe eines Tons erkannt wird daraus, dass jedem Ton eine bestimmte Strecke der Corti'schen Papille angehört. Man könnte jedoch sagen: Ein Ton von bestimmter Höhe entfaltet in der Schnecke zwei Eigensehaften:

1. er erregt die ihm eigenthümliche Strecke, d. h. die Haarzellen distalwärts bis zu einem bestimmten Punkt;

2. er erregt eine gewisse Anzahl Haarzellen mit der ihm eigenen Frequeuzzahl pro Secunde.

Daher könnte die Tonböhe bestimmt werden

a) durch die Ausdehnung der erregten Strecke, 
$\beta$ ) durch die Frequenz der Erregung;

r) durch die Ausbreitung + Frequenz.

Wir können aber die Frequenz der Erregung nicht abweichen lassen von der Frequenz des tönenden Körpers; mit jeder Zunahme der physiologisehen Frequenz nimmt aber auch die distale Fortpflanzung, die Ausbreitungsstrecke zu. Wir können also in diesem Fall zwischen $\beta$ und $\gamma$ keinen Unterschied machen. Wir können nun aber den Ausbreitungsbezirk um ein Geringes vergrössern (also $e$ Fig. 9 etwas weiter distalwärts rücken lassen), ohne die Frequenzzahl zu verändern. Der Ton wird dabei tiefer; daraus geht also hervor, dass einmal die Ausbreitungsstrecke genügt, um die Tonhöhe zu bestimmen, und dass ferner die Frequenzzahl nicht bestimmend ist. Zur Erläuterung Folgendes:

Ich habe bis jetzt angenommen, dass ein Ton von bestimmter Tonhöhe sich genau bis zu einem bestimmten Punkt fortpflanzt, weil ich das nur von der Länge der Periode habe abhängen lassen. Man könnte jedoch fragen, ob nicht die Fortpflanzungsgeschwindigkeit bei demselben Ton, wenn er stärker ist, eine etwas grössere sein müsste, weil die Curve alsdann steiler und die gebildeten Ausbuchtungen tiefer sind, also die aufgeweckten Spannungen etwas grösser. Hierauf ist $\mathrm{zu}$ antworten, dass die Amplituden erstens immer ausserordentlich klein sind, und dass sie zweitens für einen bestimmten einfachen Ton nur wenig wechseln können. Denn wir können ja gar keinen etwas starken einfachen Ton erzeugen; wenn wir das versuchen, entsteht immer ein Klang mit Grundton und Obertönen. Wir können mit andern Worten die Intensität nicht beträchtlich vergrössern, ohne dieselbe auf mehrere, bei starker Vergrösserung auf eine Unmasse von Partialtönen zu vertheilen. Es ist aber zuzugeben, dass die Amplitude eines bestimmten einfachen Tons dennoch in gewissem Grade wechseln kann: dadurch können die gebildeten Ausbuchtungen der Zona pectinata und die aufgeweckten Spannkräfte etwas grösser gemacht werden. Jetzt ist jedoch auch beim Fortschreiten der Ausbuchtung ein so viel grösseres Lymphquantum fortzubewegen; dadurch wird es möglich, dass die Geschwindigkeit der distalen Fortpflanzung sich in der Hauptsache gleich bleibt. Geben wir aber zu, dass die Geschwindigkeit bei möglichst starker Vergrösserung der Amplitude des einfachen Tons etwas grösser wird, sodass die Erregung sich auf einige Haarzellen weiter fortplanzt. 
Dann ist das im schönsten Einklang mit den Ergebnissen der Versuche von Herrn André Broca.

Dieser hat gezeigt, dass ein Ton, ohne dass die Schwingungszahl sich ändert, höher gehört wird, wenn er schwächer, und tiefer, wenn er stärker ist. (André Broca, Influence de l'intensité sur la hauteur du son; Société de Biologie 1897.) Diese Thatsache nöthigt uns also, anzunehmen, dass die physiologische Tonhöhe nur bestimmt wird durch den Abstand, worüber die Ausbuchtungen sich vom Foramen rotundum ab distalwärts fortpflanzen, und nicht von der Frequenzzahl der Erregungen. Dass sie nicht abhängen können von der Frequenzzahl, geht ferner daraus hervor, dass, wenn mehrere Töne zugleich gehört werden, die Strecke, die von einem gewissen Ton erregt wird, auch einen Theil ausmacht von den Strecken, die den verschiedenen tieferen Tönen eigen sind. Die Strecke des höheren Tons würde also mit mehreren verschiedenen Frequenzzahlen zugleich erregt werden; wir würden da unendlich viel weiter von Hause sein als bei der schönen Helmholtz'schen Resonatorentheorie. Auch kann ich mir keinen Nutzen denken von der phylogenetischen Bildung. des Schneckencanals, wenn wir einer jeden Hörzelle die Fähigkeit zuschreiben, jede beliebige Frequenz als eine bestimmte Qualität zu empfinden. Wir könnten dann mit einem primitiven Gehörorgan die grössten Musici sein.

XXXVII. Nach meiner Theorie ist, gerade wie bei der Helmholtz'schen, die Periode nur die Ursache, dass von einem bestimmten Ton bestimmte Zellen erregt werden; hierin besteht die physiologische Tonhöhe. Nach der Helmboltz'schen Theorie erregen zwei Töne theilweise die Zellen gemeinschaftlich, nach der meinigen ebenso; nach der ersten jedoch nur, wenn die Töne einander sehr nahe liegen, nach der meinigen immer. Dass dadurch das Getrennt-Hören von Tönen, die weiter von einander liegen, nicht beeinträchtigt wird, zeigt ein Rückblick auf die Curven von Fig. 10. Im Beginn der Periode des tiefen Tones $A B C D E$, wenn von diesem nur nocb kleine Ausbuchtungen gebildet sind, ist die Strecke des hohen Tones $A_{,} B_{,}, C_{,}, D_{n}, E_{n,}$, dessen Periode schon abgelaufen ist, schon bestimmt. In der zweiten Hälfte des tiefen Tons, wo von diesem am Anfang der Schnecke gar keine neuen Ausbuchtungen mehr gebildet werden, hat der hohe Ton alle Zeit ungestört zur Perception zu gelangen. Töne, die weit aus einander liegen, kommen also mit der grössten Leichtigkeit getrennt zur Per- 
ception; nach der Helmholtz'schen Theorie müsste das mit Tönen, die gerade keine Schwebungen mehr bilden, immer ebenso leicht der Fall sein. - Helmholtz fand die $4200 \mathrm{Corti}$ 'schen Bögen ausreichend für die Feinheit unserer Tonhöhenunterscheidung. Nach meiner Theorie findet man Folgendes:

Es gibt nach Retzius 12000 äussere Haarzellen. Das heisst vier Reihen von 3000 ; dies will jedoch nicht sagen: in der Länge 3000 Reihen von vier Zellen, denn die Anordnung ist so, dass immer nur zwei Zellen in jeder radialen Linie fallen; die Reihen von vier sind nicht quer, sondern ganz schief (s. die Figur).

Dadurch entstehen 6000 Querreihen von zwei Zellen. Rechnen wir die inneren Haarzellen auch mit, die wieder nicht mit den Querreihen der äusseren zusammenfallen, so kommen noch 3500 Reihen von eins hinzu, das macht zusammen 9500. Dass die verschiedenen Reihen wieder zwei an zwei gleich stark erregt werden können, ist dann noch nicht in Betracht gezogen. Auch könnte die Anordnung, wenn man die Präparate daraufhin untersuchte, vielleicht noch so sein, dass jede Zelle eine Querreihe bildet, d. h. dass nie zwei Zellen genau

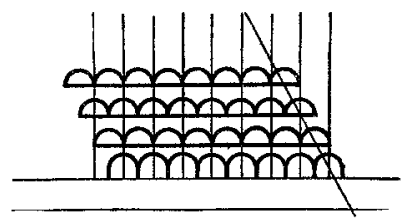

Fig. 1. in einer Querlinie liegen. Nach W aldeyer gibt es nicht 12000 , sondern 18000 , nach Krause 19800 äussere Haarzellen (Retzius, Gehörorg. d. Wirbelthiere Bd. 2). Aus der Anordnung ist ausserdem ersichtlich, dass dieselbe viel schöner einen continuirlichen Fortschritt der Tonhöhe gestattet, als wenn sie wirklich in Querreihen von vier angeordnet wären. (Man vergleiche diese Verhältnisse mit denen der percipirenden Netzhautelemente: Helmholtz, Physiologische Optik, 2. Aufl. S. 257.)

XXXVIII. Nach meiner Theorie ist das Hören einer bestimmten Tonhöhe $=$ das Erregtwerden der Haarzellen distalwärts bis zu einem bestimmten Punkt.

Ausser von dem Octavenverhältniss (Cap. XXXIII) gibt diese Theorie die einfachste Erklärung von zwei Hauptsachen:

1. Von der Thatsache, dass bei Verkürzung der Schnecke durch Wegnahme des distalen Theiles Basstanbheit eintritt. Es sind dann n. 1. die Zellen, die als Grenzpunkte der erregten Strecken die tiefen Töne bestimmen, fort; es kann wohl nicht einfacher sein. Die hohen 
Töne haben mit distalen Zellen nichts zu schaffen, die sind also nicht beeinträchtigt.

2. Von der Thatsache, dass wir die Töne in eine Reihe ordnen. Denn drei Töne von den Schwingungszahlen $n, n-10$ und $n-20$ erregen alle drei in jeder Periode die Zellen, die von $n$ ausschliesslich erregt werden; bei $n-10$ werden ausser diesen noch eine weitere Anzahl von Zellen erregt; bei $n-20$ werden die von $n+$ die für $n-10$ hinzugekommenen + noch eine weitere Anzahl Zellen erregt.

Ich kann von $n-10$ bis $n-20$ hinabgehen, obne die Strecke von $n$ dabei besonders zu markiren. Auch kann ich von $n$ bis $n-10$ hinabsteigen, ohne $n-20$ zu passiren. Ich kann jedoch nicht von $n$ bis $n-20$ hinabgehen oder von $n-20$ bis $n$ hinaufsteigen, ohne dass die Strecke von $n-10$ in der Weise des einfachen Tons ausschliesslich erregt wird, ohne dass mit anderen Worten $n-10$ passirt wird. Ich kann also von jeden drei Tönen keinen anderen als den, der mit seiner Schwingungszahl zwischen den übrigen steht, in die Mitte stellen. Dadurch ist die ganze Tonreihe bestimmt.

Dass räumliche Unterschiede in der Erregung nur im Auge und nicht in der Schnecke zu räumlichen Unterschieden in der Empfindung führen, ist selbstverständlich. Wenn beim Auge die linke Netzhauthälfte erregt wird, so wissen wir aus den in der Kindheit gehaltenen Controle mit dem Tastgefühl, dass der Gegenstand rechts ist und umgekehrt. Werden jedoch verschiedene Strecken der Schnecke erregt, so wissen wir aus der Controle mittelst des Auges und des Tastsinns, dass dennoch die Gegenstände, die die verschiedenen Strecken erregen, ain gleichen Ort sein können, und dass nach dieser Controle keineswegs die Erregung einer gewissen Strecke mit einer bestimmten Richtung des schallenden Körpers übereinkommt. Haben wir jedoch Klavierspielen gelernt, so haben wir jeden Ton mit einem gewissen Punkt der Tastatur zu combiniren uns angewöhnt; daher gibt in diesem Fall die Erregung verschiedener Schneckengegenden in gewissem Sinne zu räumlichen Unterschieden in der Empfindung Anlass, denn wir können in diesem Fall gar keine Melodie spielen hören oder uns eine solche vorstellen, ohne uns die bestimmten Stellen der Tastatur hinzuzudenken. Für die Rolle der Erfahrung beim Sehenlernen vergleiche man Helmholtz's physiologische Optik S. $731-737$ (2. Aufl.), wo die Blindgeborenen beschrieben werden, denen erst in späterem Alter das Gesicht wiedergegeben wurde. 
Für die Zerlegung eines Klangs genügt es, Lymphequanta zu finden, die in der Periode der Componenten wechseln. Ich werde hierüber nur mit zwei Worten reden, erstens, weil ich sehe, dass Herr Max Meyer, von dessen Theorie ich nur eben das Princip aus diesem Archiv habe kennen gelernt, die Curven anders vertheilt hat; zweitens, weil ich überzeugt bin, dass wir die Superpositionscurven der Lufttheilchen nicht ohne Weiteres auf die Steighügelplatte übertragen dürfen. Man betrachte nur die Figur 11, die eine Curve von zwei Tönen im Quintenverhältniss wiedergibt. Ist $O$ die Oberfläche der Steigbügelplatte, so gibt es ein Quantum $a b \times O$, das einmal in der Gesammtperiode wechselt (Differenzton); ein Quantum $b c \times O$, das dreimal wechselt (Quinte), und ein Quantum $c d \times 0$, das zweimal wechselt (Grundton). Weil während der ganzen Gesammtperiode immer noch Lymphe da ist, werden die Lymphequanta sich gleichmässig vertheilen und jedes die ihm zukommende Periode annehmen.

Nach der Helmholtz'schen Theorie wird die Superposition der einfachen Töne in die Componenten zerlegt. Nach der Bonnier'schen (und nothwendig auch nach der Ewald'schen) entsteht in der Schnecke auf's Neue eine Superpositionscurve. Herr Bonnier nimmt denn auch keine Zerlegung an. Nach meiner Theorie findet eine Zerlegung statt, daneben findet jedoch auch eine Superposition statt, die aber die Zerlegung nicht beeinträchtigt; die physische Superposition wird in eine physiologische verwandelt, die von höherer Ordnung ist, weil eben die Componenten dabei nicht verloren gehen.

Die Geräusche, die aus aperiodischen Stössen bestehen, kommen so zur Perception, dass der eine Stoss Zeit hat, die ganze Schnecke zu durchwandern, der folgende ganz in der Nähe der Fenestra rotunda bleibt $u$. s. w. Sie können daher, wenn sie absolut aperiodisch sind, auch keine bestimmte Tonhöhe haben. Je öfter einige einander auffolgende Stösse um dieselbe Periode von einander $a b$ stehen, desto öfter wird dann und wann eine Tonhöhe unterschieden werden; diese ist aber dann immer noch sehr wechselnd. Dies verhindert jedoch nicht, dass in jedem Geräusch ein oder mehrere Töne fortwährend anwesend sein können. 